\title{
Peritoneal Tuberculosis: Challenging to Diagnose, But Curable
}

\author{
Harada $\mathbf{Y}^{1^{*}}$, Kunimura $\mathrm{T}^{2}$, Sasaki $\mathrm{Y}^{3}$, Oonishi $\mathrm{T}^{3}$, and Takenoue $\mathrm{T}^{3}$ \\ ${ }^{1}$ Department of General Internal Medicine, Yamato Tokushukai Hospital \\ ${ }^{2}$ Department of Pathology, Yamato Tokushukai Hospital \\ ${ }^{3}$ Department of Surgery, Yamato Tokushukai Hospital
}

*Corresponding author: Harada Y, Division Head, Department of General Internal Medicine, Yamato Tokushukai Hospital 4-4-12 Chuo, Yamato Kanagawa, 242-0021, Japan, Tel: +81-46-264-1111; E-mail: yu_harada@ yth.or.jp

Received: February 14, 2020; Accepted: February 25, 2020; Published: March 04, 2020

\begin{abstract}
A 79-year-old woman was admitted due to ileus. Test laparotomy revealed numerous nodules with calcification disseminated on the surface of peritoneum, omentum, small intestine, and mesentery, which resembled peritoneal dissemination of cancer. Histopathological examination revealed that the nodules were consisted of caseating granuloma, and acid-fast bacilli were detected. Four-drug therapy for tuberculosis was initiated, and patient completely recovered. Peritoneal tuberculosis was proven to be curable despite difficulty of diagnosis. Even though the incidence is decreasing every year, latent tuberculosis should always be taken into consideration for elderly patients. If a patient shows ileus with unknown cause, peritoneal tuberculosis cannot be ruled out even if the sputum culture or T-spot are negative.
\end{abstract}

Keywords: Peritoneal tuberculosis; Ileus; Laparotomy

\section{Introduction}

Tuberculosis is rare in developed countries; however, it has been a serious infection in Japan for over 100 years. After invention of multiple-drug therapy, the mortality rate was significantly decreased. Extrapulmonary tuberculosis such as peritoneal tuberculosis is also curable with multiple-drug therapy, but is challenging to diagnose.

\section{Case Report}

A 79-year-old woman with a history of pleuritis was admitted to our hospital because of frequent vomiting and abdominal distention. She did not have abdominal pain. The blood test showed increased white blood cell counts of $13,300 / \mu \mathrm{L}$, and slightly elevated CRP level of $1.639 \mathrm{mg} / \mathrm{dL}$. Computed tomography (CT) of the abdomen and pelvis revealed severe

Citation: Harada Y, Kunimura T, Sasaki Y, et al. Peritoneal Tuberculosis: Challenging to Diagnose, But Curable. Clin Case Rep Open Access. 2020;3(1):140. 
dilatation of small and large intestine. A diagnosis of small-bowel obstruction was suspected, but the cause was unclear because she did not have a history of abdominal surgery nor constipation. After inserting nasogastric tube, nausea was resolved. On the third hospital day, gastrointestinal series were operated (FIG. 1A). Contrast medium Gastrographin flowed slowly but smoothly, and the patient had bowel movement in the evening. Then she developed constipation. Six days later, contrast $\mathrm{CT}$ of the abdomen revealed diffuse enlargement of the intestine and retention of Gastrographin which was supposed to be discharged days ago (FIG. 2). On the tenth hospital day, test laparotomy was performed. Numerous nodules were found on the surface of the peritoneum and the omentum (FIG. 3). A part of the omentum adhered to the abdominal wall, which was difficult to detach. The nodules were disseminated on the whole small intestine, mesentery, and abdominal wall. Peritoneal dissemination due to carcinomatous peritonitis was suspected to be the cause of the paresis of intestine. However, histopathological examination revealed that the nodules were consisted of caseating granuloma (FIG. 4A). An acid-fast stain revealed a few acid-fast bacilli (FIG. 4B). The diagnosis of peritoneal tuberculosis was suspected. During surgery, peritoneal tuberculosis was not listed in differential diagnosis, thus the specimen was not cultured. The surgical observation was very similar to carcinomatous peritonitis, and the patient's pre-surgical tests for tuberculosis were all negative, which thus did not point to peritoneal tuberculosis.

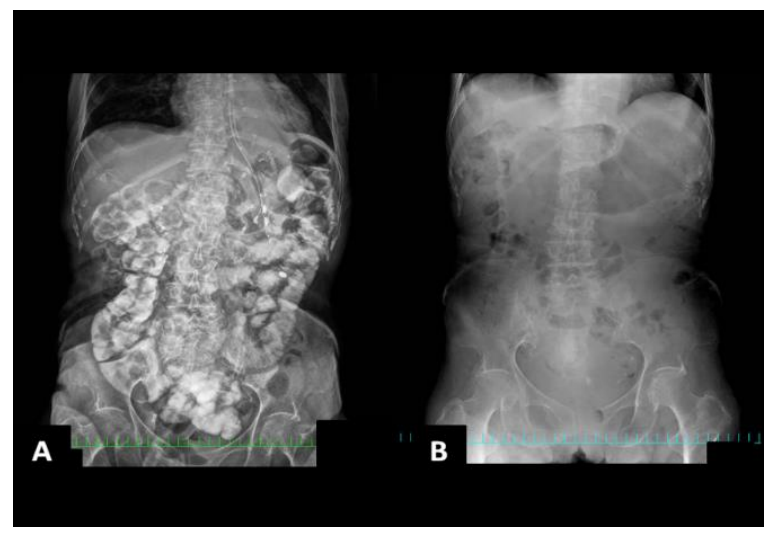

FIG. 1. Abdominal X-ray findings. A: Gastrointestinal series revealed no occlusion in the intestine, but retention of contrast medium at 2 hours shows slow movement. B: Intestinal gas disappeared in 9 weeks after medication started.

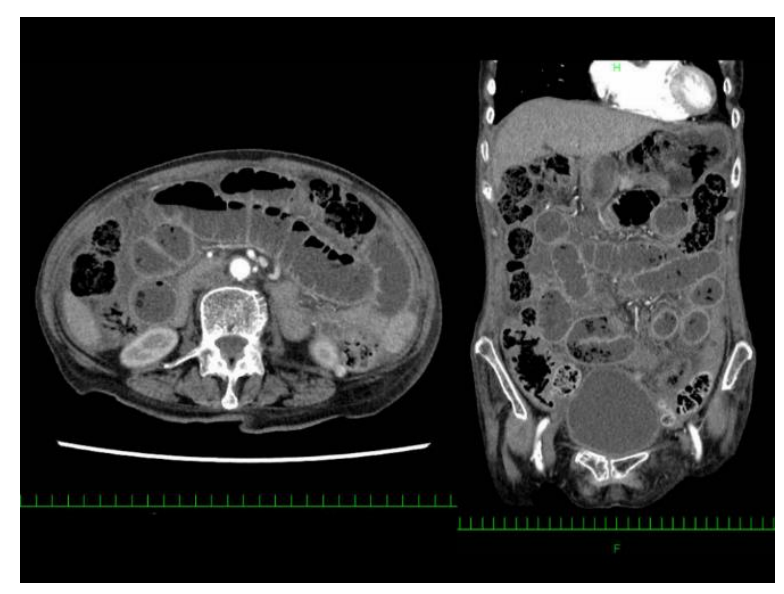

FIG. 2. Abdominal CT. Liquid retention is observed in the intestine. 


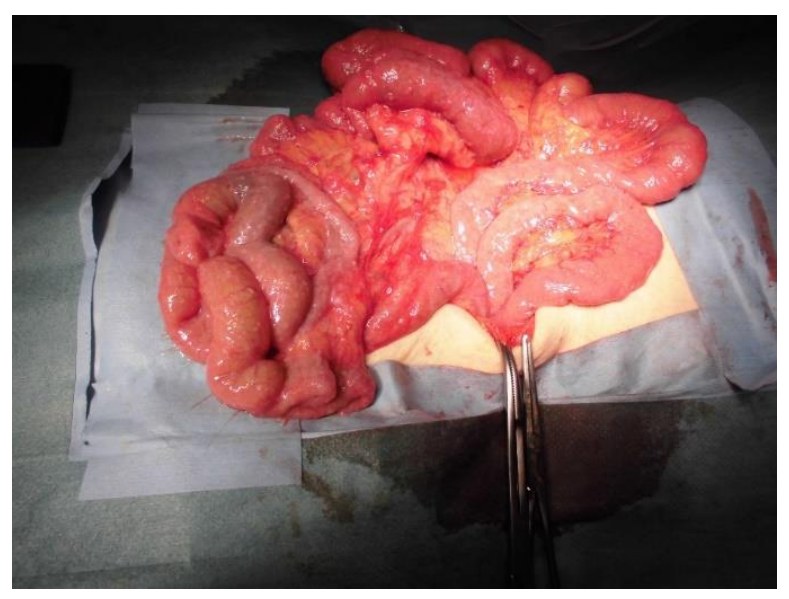

FIG. 3. Laparotomy. Nodules are disseminated on the surface of small intestine, mesentery, and abdominal wall.

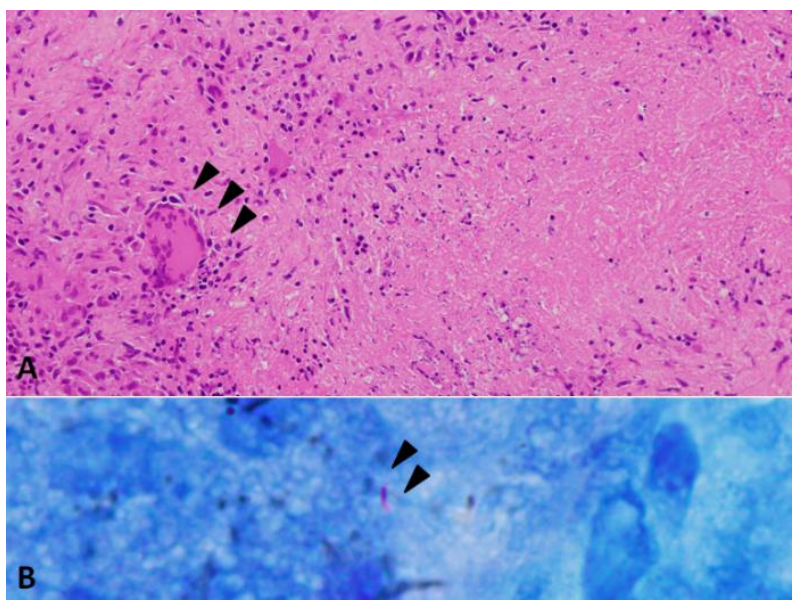

FIG. 4. Biopsied nodule on mesentery. A: Hematoxylin-eosin stain shows epithelioid granulomas with caseous necrosis. Langhans giant cells are also shown (arrowheads). B: Acid-fast stain reveals an acid-fast bacterium stained in pink (arrowheads).

Six months before the current admission, the patient was admitted to our hospital due to pleuritis (FIG. 5). Antibiotics were not effective. As the patient had calcification in the upper lobe of the lungs, pleural tuberculosis was suspected. However, acid-fast stain was negative for sputum and gastric fluid. The pleural effusion was exudate, but Adenosine deaminase (ADA) level was only $14.6 \mathrm{IU} / \mathrm{mL}$ which was unlikely for pleural tuberculosis. As CEA level of pleural effusion was elevated to $14.1 \mathrm{ng} / \mathrm{mL}$ (serum CEA $6.4 \mathrm{ng} / \mathrm{mL}$ ), a diagnosis of carcinomatous pleuritis was suspected. Cytology was negative (Class II), and the patient did not wish to have further evaluation for cancer. As the amount of pleural effusion was only $1 / 2$ of the left lower lobe, the patient was discharged for watchful waiting at a nursing home. The pleural effusion decreased naturally over 6 months (FIG. 6).

During the current admission, acid-fast stain and culture were repeated for sputum ( 3 consecutive days), gastric fluid, blood, and stool, all of which were negative. T-SPOT.TB was positive. Once the diagnosis of tuberculosis was confirmed, four-drug 
therapy with rifampin, isoniazid, pyrazinamide, and ethambutol was started. Panthenol $200 \mathrm{mg}$ /day was administered daily, but the patient didn't have bowel movement without using enema every 3 days. Oral intake was prohibited, and intravenous hyperalimentation was used for 2 months until the patient had natural bowel movement. The patient was able to take medicine orally without vomiting even though she reported nausea for the first few weeks.

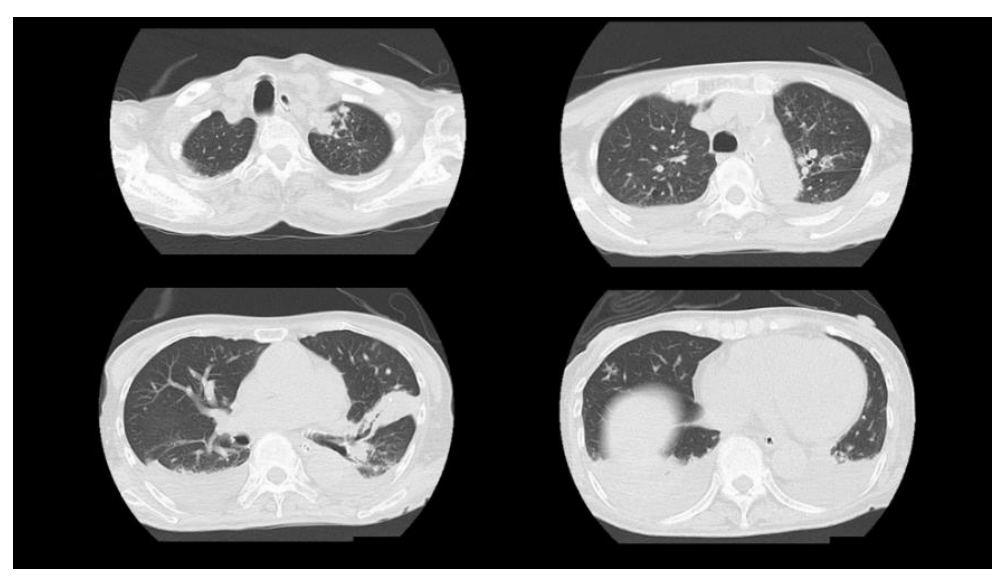

FIG. 5. Chest CT scan showing pleuritis. Pleural effusion is shown on both sides. Consolidation is observed in left lower lobe. Cavitated nodule is observed in left upper lobe, which was formed by old tuberculosis.

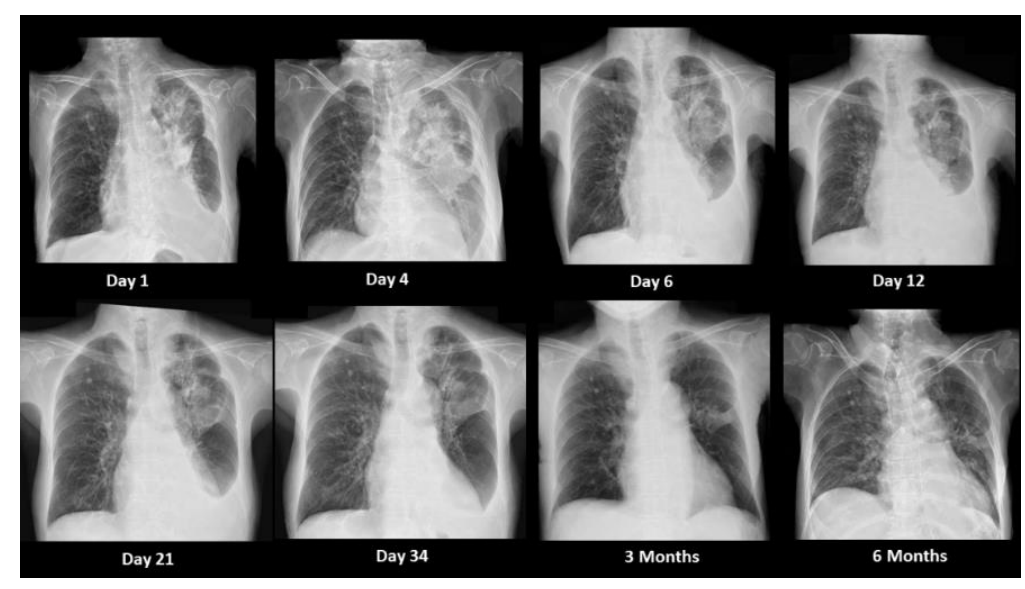

FIG. 6. Chest X-ray from the first day of admission for pleuritis. Pleural effusion disappeared over 6 months when the patient was admitted for ileus.

Seven weeks after starting medication, abdominal X-ray still showed remarkable colon gas retention. Kampo medicine named Daikenchuto $7.5 \mathrm{mg}$ /day was started to resolve constipation. Eight weeks after starting medication, oral intake was started and 4-drug therapy was switched to 2-drug therapy with rifampin and isoniazid. The patient had natural bowel movement in one week, and abdominal X-ray showed no gas retention (FIG. 1B). She was able to eat regular meal, and was able to sit on a wheelchair as she was before admission. She was discharged to the nursing home in good condition to continue 2-drug therapy for 4 months. 
www.yumedtext.com | March-2020

\section{Discussion}

Tuberculosis is more prevalent in Asian countries than Western countries. The Tuberculosis Surveillance Center of Japan reported that the total number of patients in 2018 was 15,590, compared to that of 16,789 in 2017. The number of peritoneal tuberculosis was 148 in 2018 and 178 in 2017 [1]. They also reported that the success rate of treatment was 68.2\% and mortality rate was $22.5 \%$ in 2018 [1]. Thus peritoneal tuberculosis is rare compared to lung tuberculosis, and the number of patients are decreasing every year. The mortality rate is also decreasing, and tuberculosis is a curable disease today.

In January 2018 the committee for treatment, the Japanese Society for Tuberculosis announced the latest guideline for treatment of tuberculosis [2]. For the primary treatment, they recommend 4-drug therapy with rifampin, isoniazid, pyrazinamide, and ethambutol for 2 months followed by 2-drug therapy with rifampin and isoniazid for 4 months. The patient had calcification in her lungs suggestive of prior tuberculosis, but she didn't have a history of treatment for tuberculosis. Thus she was treated with the regimen for the primary treatment, which she tolerated well. As tissue culture was not obtainable, the standard treatment regimen was chosen. All the symptoms disappeared and the laboratory tests and X-ray were normal at 8 weeks of treatment, but she continued 2-drug therapy for 4 months to prevent relapse.

Making a diagnosis for peritoneal tuberculosis is difficult, as it does not have specific clinical features. Averbukh LD et al. reported in their review that commonly described symptoms and signs among 1725 cases were abdominal pain $75 \%$, weight loss $53.0 \%$, fever $69 \%$, ascites $62.0 \%$, abdominal distension $60.0 \%$, abdominal mass $34.0 \%$, and abdominal tenderness $49.0 \%$ [3]. This confirms the disease being not localized but spread to the entire intestine and mesentery. Our case demonstrated abdominal distension, but no pain nor tenderness.

The gold standard for definitive diagnosis is peritoneal biopsy and subsequent pathological or microbiologic confirmation [3]. Tissue culture is essential. Laparoscopy is preferred over open surgery. However, tuberculosis is not always detected with acid-fast stain of the specimen obtained from surgery or laparotomy. The ascitic fluid test is frequently performed, but acidfast stain is generally insensitive. Manohar A et al. [4] analyzed 145 patients and reported that no patient had detected acidfast bacilli in aspirated ascites, but they were found in $73.8 \%$ of the biopsy specimen, and that granuloma with caseation was found in all of the specimen [4]. A recent review article reported that laparoscopy with directed biopsy provides a rapid and correct diagnosis in 76-100\% of cases [5]. CA125 and ADA in ascites are said to be useful for diagnosis, but none of them are more reliable than biopsy [3,5,6]. Our case showed only small amount of ascites, and presented with ileus which necessitated urgent resolution, thus laparotomy was performed rather than laparoscopy, which led to successful diagnosis and treatment. As ascites were not collected during surgery, CA125, ADA, and culture of ascites were not tested.

The most common cause of peritoneal tuberculosis is reactivation of latent tuberculosis foci in the peritoneum established via hematogenous spread from a primary lung focus [7]. Our case had pleuritis 6 months prior to the current admission, and also had suspective old tuberculosis foci in her lungs. She likely had lung tuberculosis when she was young and recovered without medication, which may have caused pleuritis and peritonitis. The cause of pleuritis was undetectable in the previous admission, but it probably was also tuberculosis because pleural effusion completely disappeared after 4-drug therapy for tuberculosis. 
This is a case of peritoneal tuberculosis which presented with the symptom of ileus after pleuritis and was diagnosed by test laparotomy and successfully treated with 4-drug therapy. It resembled cancerous peritonitis, but histopathological examination successfully detected characteristic granuloma with central necrosis and acid-fast bacilli. If a patient shows calcification in the lungs, latent tuberculosis should be always considered.

\section{REFERENCES}

1. The Tuberculosis Surveillance Center. [http://www.jata.or.jp/rit/ekigaku/en/statistics-of-tb/]

2. The committee of treatment, the Japanese Society for Tuberculosis. Revision of standard treatment for tuberculosis2019 (in Japanese). Kekkaku. 2018;93(1):61-8.

3. Wu DC, Averbukh LD, Wu GY. Diagnostic and therapeutic strategies for peritoneal tuberculosis: A review. J Clin Transl Hepatol. 2019;7(2):140-8.

4. Manohar A, Simjee AE, Haffejea AA, et al. Symptoms and investigative findings in 145 patients with tuberculosis peritonitis diagnosed by peritoneoscopy and biopsy over a five-year period. Gut.1990;31(10):1130-2.

5. Ahmed Khan FY. Peritoneal tuberculosis: Advances and controversies. Libyan J Med Sci. 2018;2(1):3-7.

6. Kishimoto K, Mishiro T, Mikami H, et al. Measurement of adenosine deaminase in ascitic fluid contributed to the diagnosis in a case of tuberculosis peritonitis. Case Rep Gastroenterol. 2019;13(2):299-304.

7. Mehta JB, Dutt A, Harvill L, et al. Epidemiology of extrapulmonary tuberculosis. A comparative analysis with preAIDS era. Chest. 1991;99(5):1134-8. 